\title{
Design and Characterization of RNA Nanotubes
}

\author{
Jaimie Marie Stewart, ${ }^{\dagger}$ Cody Geary, ${ }^{\ddagger}, \perp$ and Elisa Franco ${ }^{*,},, \| \odot$ \\ ${ }^{\dagger}$ Department of Bioengineering, University of California at Riverside, Riverside, California 92521, United States \\ ${ }^{\star}$ Department of Bioengineering, California Institute of Technology, Pasadena, California 91125, United States \\ ${ }^{\perp}$ Interdisciplinary Nanoscience Center, Aarhus University, Aarhus C 08000, Denmark \\ ${ }^{\S}$ Department of Mechanical Engineering, University of California at Riverside, Riverside, California 92521, United States \\ "Department of Mechanical and Aerospace Engineering, University of California, Los Angeles, California 90095, United States
}

\section{Supporting Information}

\begin{abstract}
RNA is a functionally rich and diverse biomaterial responsible for regulating several cellular processes. This functionality has been harnessed to build predominately small nanoscale structures for drug delivery and the treatment of disease. The understanding of design principles to build large RNA structures will allow for further control of stoichiometry and spatial arrangement drugs and ligands. We present the design and characterization of RNA nanotubes that selfassemble from programmable monomers, or tiles, formed by five distinct RNA strands. Tiles include double crossover junctions and assemble via single-stranded sticky-end domains. We find that nanotube formation is dependent on the intertile crossover distance. The average length observed for the annealed RNA nanotubes is $\approx 1.5 \mu \mathrm{m}$, with many nanotubes exceeding $10 \mu \mathrm{m}$, enabling the characterization of RNA
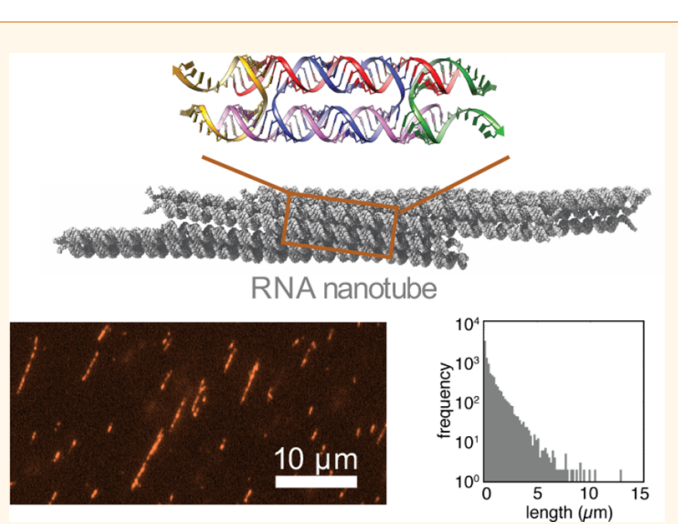
nanotubes length distribution via fluorescence microscopy. Assembled tubes were observed to be stable for more than $24 \mathrm{~h}$, however post-annealing growth under isothermal conditions does not occur. Nanotubes assemble also from RNA tiles modified to include a single-stranded overhang (toehold), suggesting that it may be possible to decorate these large RNA scaffolds with nanoparticles or other nucleic acid molecules.
\end{abstract}

KEYWORDS: RNA, nanotechnology, nucleic acids, self-assembly, nanotubes

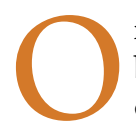

ne of the goals in nucleic acid nanotechnology is to build programmable scaffolds of scalable size and complexity. While small, compact structures are very useful to build nanomachines and drug delivery vectors, large scaffolds are needed to undertake challenges such as controlling the stoichiometry and spatial organization of multivalent drugs, ligands, and enzymes. Precise spatial control of ligands, for instance, can yield improved mass transport and efficiency, as demonstrated with large DNA scaffolds designed to include target binding sites at prescribed locations. ${ }^{1-3}$ Although DNA nanotechology has demonstrated a wide variety of assembly sizes, patterns, and packing, ${ }^{4}$ RNA is emerging as an even more versatile molecule, ${ }^{5-8}$ capable of the standard Watson-Crick pairing as well as and noncanonical base pairing that can yield a variety of structural motifs with functional properties that include: catalysis, gene regulation, aptamers, siRNA, miRNA, and ribozymes. Thus, RNA has many advantages over DNA for in vivo biomedical applications.

RNA nanostructures exceeding thousands of nucleotides have been recently demonstrated using few, very long RNA strands that fold into the desired structures exploiting local domain interactions. ${ }^{6,10}$ This approach is advantageous in view of future in vivo transcription and folding of such structures, but it is costly and presents a low yield for the purpose of in vitro production of large scaffolds. There are two general challenges that make it problematic to use long RNA strands to build large nanoscale assemblies. First, RNA structure is more difficult to predict and design when compared to DNA, primarily because RNA molecules present a number of thermostable noncanonical interactions and conformations; thus, the secondary structure can be significantly affected by small mutations introduced, for instance, for functionalization purposes. Second, the production of long RNA molecules with artificial sequences is problematic with any methods. ${ }^{11-14}$ Solid-phase synthesis of long RNA strands is particularly expensive and presents a low yield of correct products. Likewise, in vitro transcription of long strands of RNA is

Received: December 12, 2018

Accepted: April 9, 2019 
A
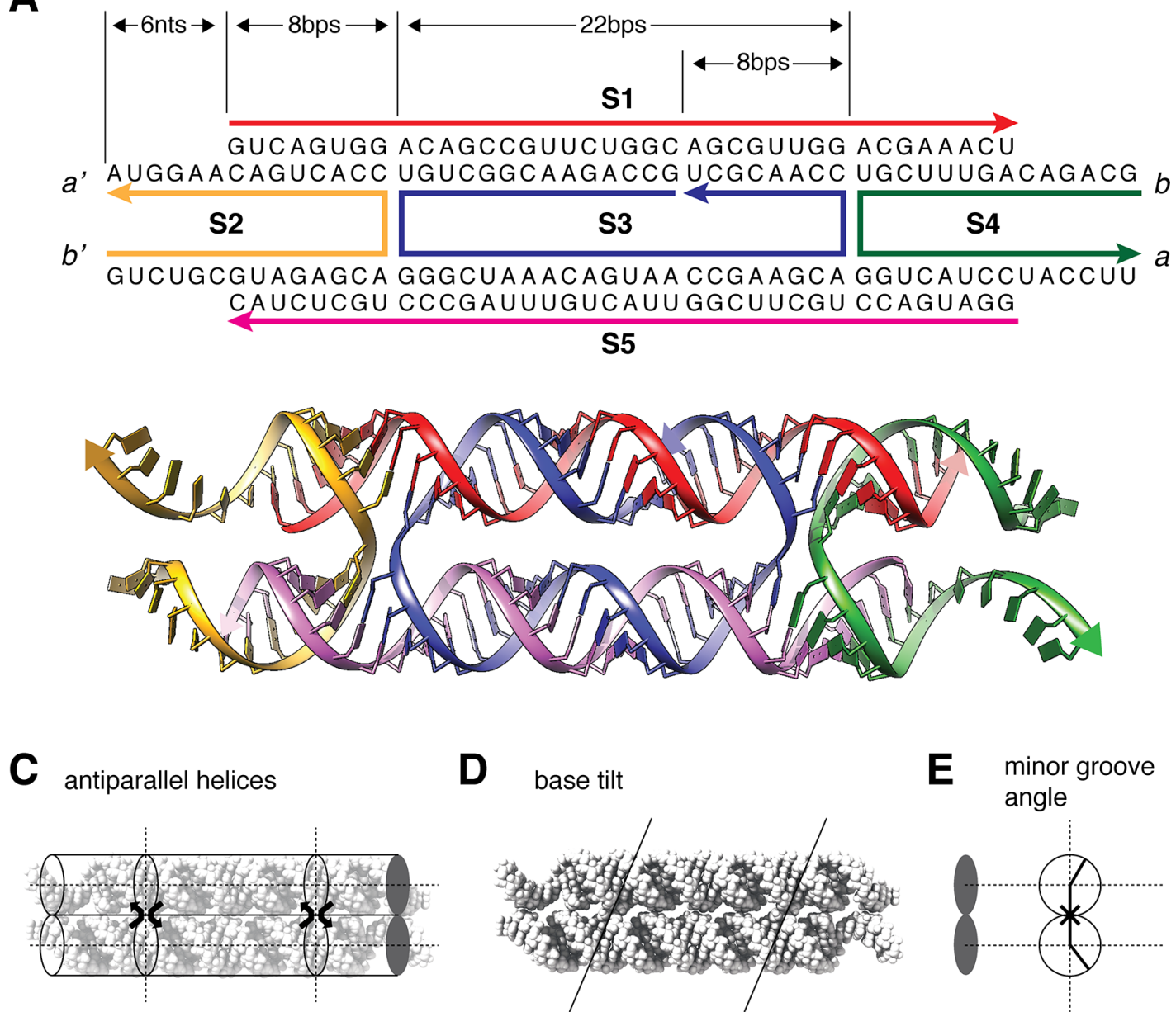

D base tilt

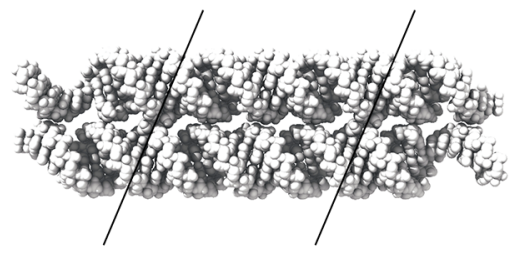

$\mathbf{E}$

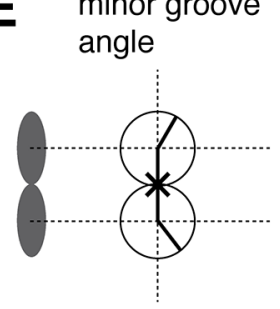

$\mathbf{F}$

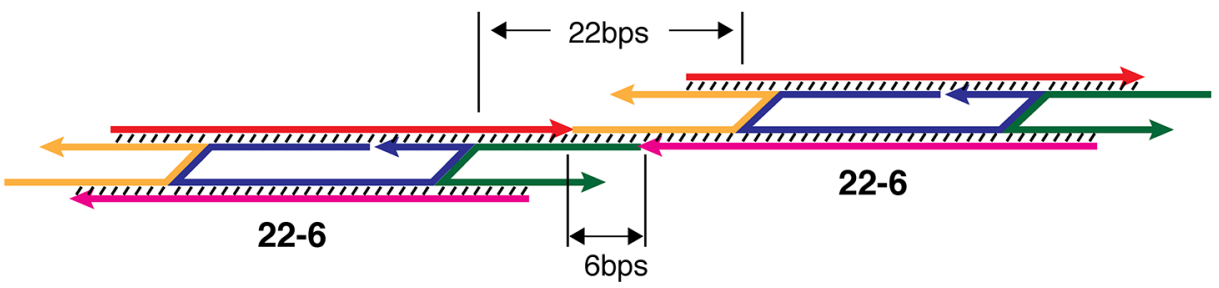

Figure 1. We designed an RNA double-crossover tile expected to form curved lattices. (A) Two-dimensional schematic and sequences of our RNA DAE-E tile; we focus on a variant in which the inter- and intratile crossover distance is 22 base pairs. (B) Three-dimensional rendering of the DAE-E RNA tile. (C) Scheme highlighting the antiparallel nature of the helices included in the tile. (D) The tilt of RNA bases is coherent across the two helices of the tile and induces a twist in tile assemblies. (E) Schematic of the tile cross-section that emphasizes the minor groove angle at which distinct tiles should interact, forming curved lattices. (F) Illustration of two RNA tiles interacting through their sticky ends; the tilt of RNA bases adds an offset in the direction of the coaxial stacking of tiles.

expensive because it still requires the assembly of long artificial DNA strands to build the corresponding templates, which may require additional purification steps.

It is simpler and more cost-effective to design and build large RNA structures assembling from many short RNA molecules that can be transcribed from inexpensive short DNA templates with high yields. Using this method, large amounts of RNA can be produced in just a day from a minuscule amount of DNA template for subsequent assembly; structure formation could be even obtained with a one-pot approach. ${ }^{7}$ A variety of structured RNA lattices has been built with short RNA sequences forming "tiles" (tecto RNAs) that interact and assemble via kissing loops, ${ }^{15,16}$ yet these structures are orders of magnitude smaller than those obtained with DNA tiles interacting via single-stranded "sticky ends. ${ }^{17,18}$ With this in mind, we attempted to produce a system where large-scale RNA structures assemble cooperatively from multiple copies of smaller RNA strands, looking to both nature and DNA nanotechnology for inspiration. We aimed at building filamentous assemblies which are a common motif in nature; for example, the cytoskeleton is comprised of many types of filamentous components such as actin, or tubulin among others, that form thread-like structures that function to direct cellular motility and transport. Many variants of artificial DNA nanotubes have been demonstrated and characterized, ${ }^{17-20}$ however nanotubes of a similar size have not been obtained with RNA. 
A
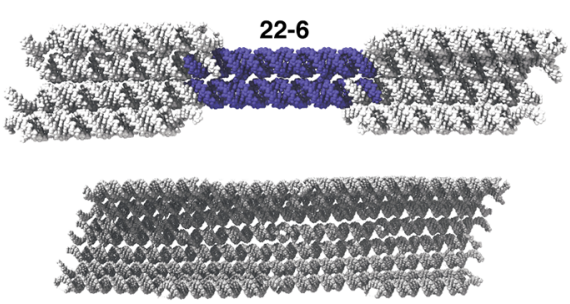

B

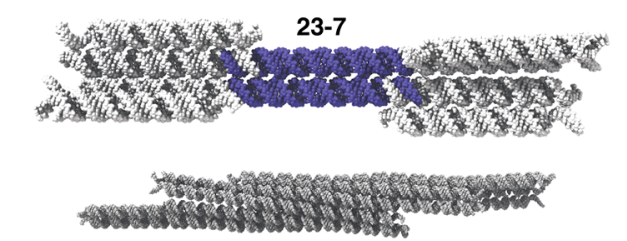

C

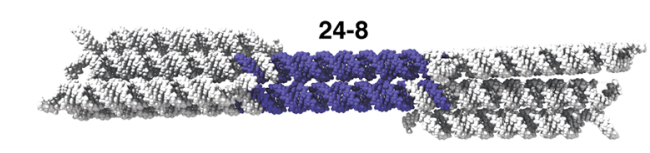

D

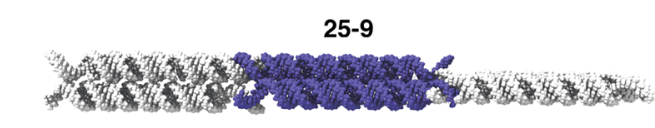

E

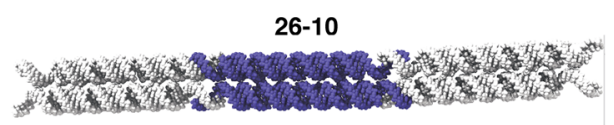

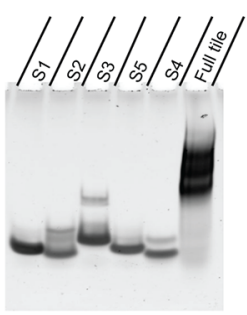
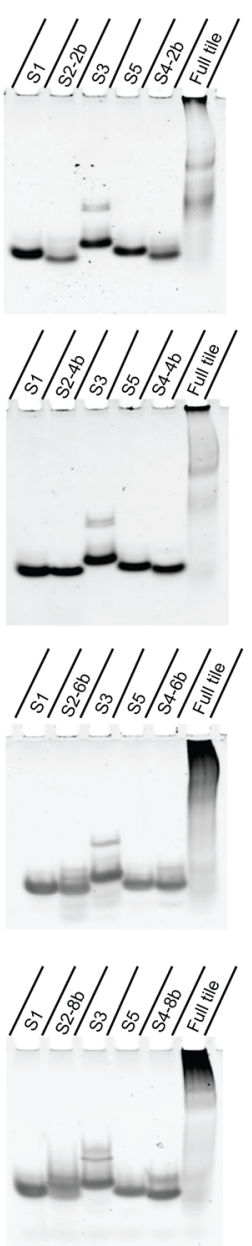
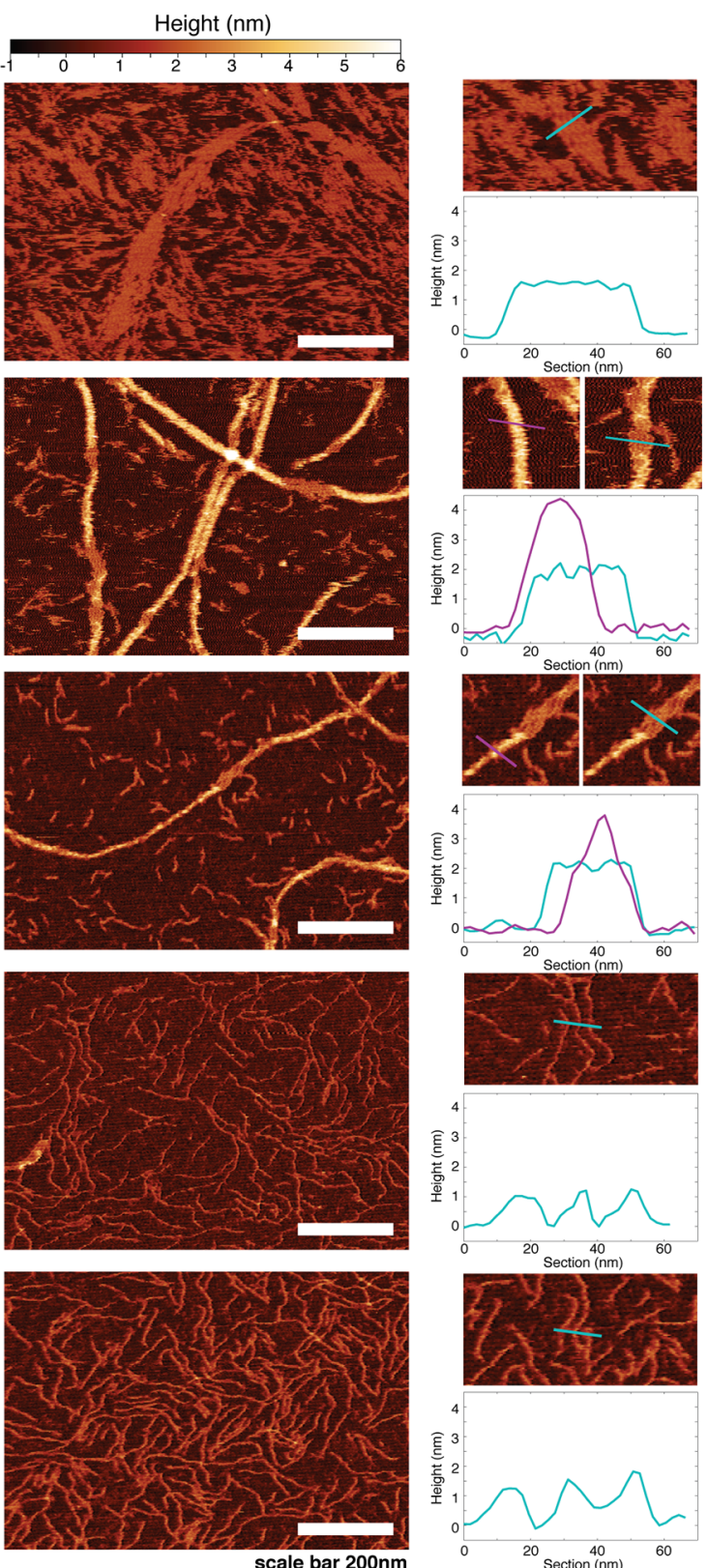

Figure 2. Nanotube formation depends on the intertile crossover distance. We provide an overview of modeling and experimental results evaluating the assembly of five DAE-E tile variants. (A-E) Left column shows the three-dimensional models of tile interactions. In all models, we highlighted in blue a single example tile for each variant, and the gray tiles are representative of how the adjacent neighboring tiles are expected to assemble based on the intertile crossover distance, resulting in either a flat two-dimensional lattice, a three-dimensional nanotube, or two-dimensional filamentous assemblies. The left middle column shows $10 \%$ native PAGE of the tile components and assemblies. The right middle column shows example AFM images of the assemblies, the scale bar is $200 \mathrm{~nm}$. The right column shows representative height measurements of structures along with the corresponding AFM images. Blue and purple lines mark the height measurement segment on the imaging area. Additional large scan area AFM images are shown in Figure S8.

We demonstrate the assembly of micron-sized RNA nanotubes assembled from a set of five short single-stranded RNA transcripts. The RNAs are easily prepared in high yield from short DNA templates. With thermal annealing these RNA strands hybridize into tiles that then assemble via singlestranded sticky ends into quaternary structures and are intended to be structurally equivalent to tiles formed from DNA strands that yield nanotubes and lattices. ${ }^{17}$ Thus, each tile is formed when four RNA strands assemble in a complex around a central RNA strand (Figure 1A). The tiles bind to each other by canonical base pairing of single-stranded domains (sticky ends), a simple mode of programmable interaction for assembling RNA, in contrast with other assembly motifs involving tertiary folds such as kissing loops, receptors, or aptamers. ${ }^{6,15,21-23}$ Here we extend the tail-tail connections described in ref 15 to also include the $5^{\prime}$ end head-to-head contacts and so refer to these interactions of RNA generally as sticky ends. The exclusion of secondary and tertiary structure motifs allows these RNAs to be prepared by thermodynamic annealing, under similar conditions to DNA assemblies. The lack of tertiary motifs and strong magnesium binding sites on the RNA may help to decrease the chemical 
lability of the RNA strand during the course of the thermal annealing ramp. ${ }^{24,25}$

We investigate the effect of simultaneously modulating the intertile crossover distance and sticky-end length in RNA tiletile assemblies built with the same core tile. These design variants assemble into a diversity of a structures ranging from flat sheets to tubes to one-dimensional filaments by changing only the geometry of the interconnection between tiles. We demonstrate that some of our tile variants yield nanotubes with an average length of $\approx 1.5 \mu \mathrm{m}$, which can be imaged with epifluorescence microscopy. Likewise, we also demonstrate that the sticky-end interactions can be extended into toehold binding sites and still assemble into tubes, and so may be potentially useful for linking to other to molecules such as siRNA and miRNA within RNAi applications, aptamers, or DNA or RNA molecules linked with organic or non-organic ligands. We foresee that these nanotubes will be useful as molecular scaffolds for biomedical and drug-delivery applications $^{8}$ as well as structural or mechanical components within artificial cells. ${ }^{26}$ Further, sticky-end interactions could potentially be controlled via strand displacement reactions, as recently demonstrated in DNA tiles, ${ }^{27,28}$ to build reconfigurable RNA scaffolds.

\section{RESULTS AND DISCUSSION}

To obtain self-assembling tubular structures from nanoscale RNA monomers, the geometry of the monomers, as well as the symmetry of their interactions, should be compatible with the formation of a curved lattice. We hypothesized that DNA tiles optimized by Rothemund et al. ${ }^{17}$ to assemble into DNA nanotubes could be readily adapted to the characteristics of RNA's A-form helices, while preserving the correct interaction geometry for tubular assemblies. These tiles consist of two double helices held together by two Holliday junctions ans thus are termed double crossover tiles; the orientation of the strands through the crossover is antiparallel. Tiles interact via complementary single-stranded domains, or sticky ends. In this particular design, the crossovers within each tile, as well as between interacting tiles, are spaced by two full helical turns. Hence they are known as DAE-E tiles (double crossover, antiparallel, even number of intra- and intertile half-turns between crossovers). ${ }^{17,29}$

We designed an RNA DAE-E tile (Figure 1A,B) in which five unique RNA strands ( $\mathrm{S} 1, \mathrm{~S} 2, \mathrm{~S} 3, \mathrm{~S} 4$, and S5) bind via Watson-Crick complementary domains. Strands S2 and S4 include single-stranded sticky ends that extend outward from the tile and are designed to promote the connection of tiles into structured assemblies. The intratile crossover distance is constrained in all designs to be 22 base pairs (bp) exactly, to accommodate for two helical turns of RNA; by comparison, 21 bp are required for the equivalent DNA DAE-E tiles. Strands S2, S3, and S4 participate in the formation of crossovers within the tile, while the backbone of strands $S 1$ and S5, as it extends from the tile, is meant to interact coaxially with neighboring tiles upon assembly (Figure $1 \mathrm{~F}$ ).

If we consider the tile to be roughly planar, then the angle at which two tiles assemble will be decided by a combination of the intertile spacing and the minor-groove angle of the helix. This geometry for tubular assemblies has been well characterized for the DNA tiles ${ }^{17}$ upon which our RNA design is based. In the DNA arrays, the intertile spacing was set to 21 bp ( 2 helical turns, and equivalent to the intratile crossover spacing), upon which DNA tiles interact with an angle of roughly $150^{\circ} \pm 10^{\circ}$, in line with observed nanotubes with a diameter of 4-9 tiles.

Although the minor groove angle of RNA (A-form) is similar to that of DNA (B-form), the geometry of two interacting RNA tiles is also influenced by the $19^{\circ}$ inclination of the base pairs in RNA (Figure 1D,E) as well as the different helicity (11 bp/turn vs $10.5 \mathrm{bp} /$ turn). Interestingly, the base inclination of RNA adds an offset in the direction of the coaxial stacking of tiles (Figure $1 \mathrm{~F}$ ), and consequently this results in a twist in the lattice that constrains the way it can close into a tube. (In comparison, the base inclination of DNA is too small to result in naturally twisted DNA nanotubes.) These considerations suggest that an intertile crossover distance that is not exactly two helical turns (as in the original DNA DAE-E design) may be required to achieve the self-assembly of nanotubes.

To identify the optimal intertile crossover distance for the formation of tubular structures, we started from the "nominal" RNA DAE-E tile shown in Figure 1A,B and designed four additional tile variants in which the intertile crossover distance is increased from 22 to 26 bases. In each variant, we exclusively elongated the single-stranded sticky-end domains, leaving all of the double-stranded domains of the tile unchanged. Hence, strands S1, S3, and S5 are the same for all tile variants considered here, unless otherwise noted. We named the tile variants by the intertile crossover distance and sticky-end length; for example, in the tile in Figure 1A, the intertile crossover distance is 22 bases and the sticky-end length is 6 bases, so this tile variant is named 22-6. An overview of the variants we consider is in Figure 2.

We characterized the self-assembly products of each tile variant using both atomic force microscopy (AFM) and nondenaturing polyacrylamide gel electrophoresis (native PAGE), and to support our experimental observations, we also generated three-dimensional models of putative assembly products (SI Section 10). To produce RNA assemblies, five RNA strands of each variant were individually transcribed from linear templates and gel purified. Then, the strands required to build each tile variant were mixed and slowly annealed for $24 \mathrm{~h}$.

Three-dimensional modeling of 22-6 (Figure 2A) suggests that the tiles are able to arrange into a highly ordered, rectilinear grid. The predicted curvature of the assembly should facilitate tubes to form. The AFM images in Figure 2A show that this tile variant yields rectilinear lattices with a low aspect ratio as predicted, but no tubular structures were observed. The presence of few thick bands in native PAGE suggests that these tiles may nucleate and form only very small lattices in solution, ${ }^{7}$ and that their growth into larger rectilinear lattices could be mica-assisted. AFM height analysis indicates that 22-6 assembles into flat structures whose thickness is comparable to an RNA double helix. The observation of large numbers of small tile patches by AFM, and also tile-sized assemblies observed by gel, suggests that the tiles rapidly nucleate into the small complexes which then do not further grow into nanotubes. We hypothesized that a variant of the tile with different tail-tail connection geometry might lead to a more stable nanotube formation.

We generated a series of structures with increasing tile-tile spacing by exclusively increasing the number of nucleotides in the sticky-end connections between tiles, which changes the twist in the connection geometry by $\approx 32.7^{\circ}$ increments $(1 / 11$ of a full rotation). By modeling the elongated connections between tiles as rigid helices, our model of 23-7 predicts an 
A

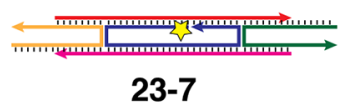

23-7

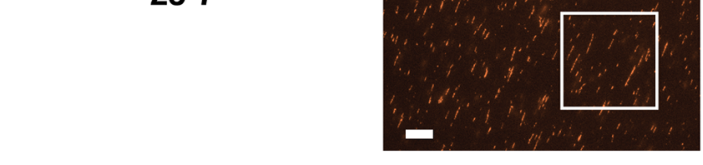

B

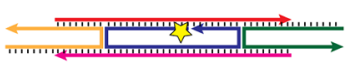

24-8

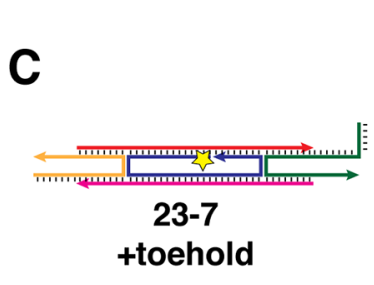

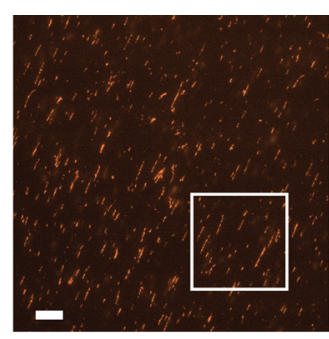
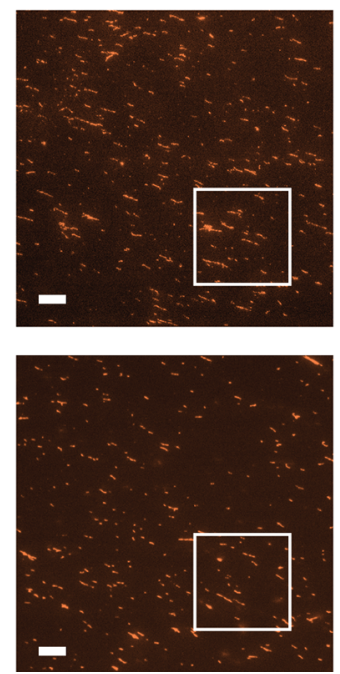
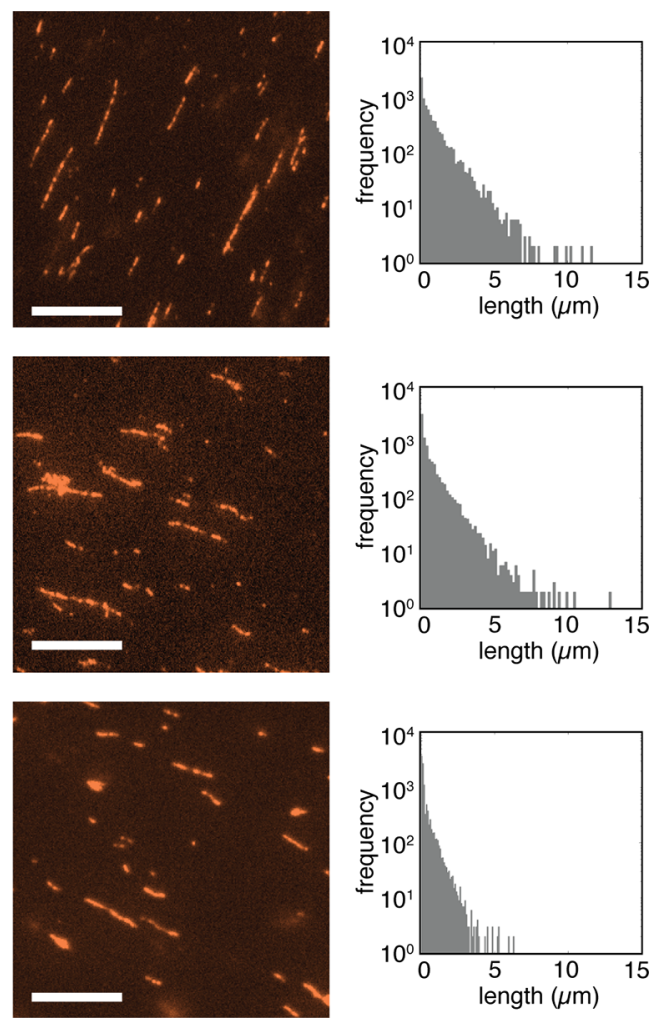

Figure 3. Characterization of RNA nanotubes using epifluorescence microscopy. (A-C) Left: Scheme of each tile variant, in which the strand S3 was labeled with cy 3 fluorophore at the $5^{\prime}$ end (shown as a yellow star). Middle: Representative fluorescence microscopy images of the nanotubes assembled from the corresponding tile variant. Right: Normalized histograms of the nanotube length after annealing. Scale bar is $10 \mu \mathrm{m}$.

overall twist to the resulting assemblies, if constrained to form a nanotube (Figure 2B, variant 23-7). Further increasing the length of the sticky-end connection increases the twist between the tile-tile connections (Figure 2C, variant 24-8), but in full structural modeling, the $23-8$ assembly as a tube was not possible due to the large number of predicted steric clashes after just one layer of tiles are joined. AFM images of annealed 23-7 and 24-8 variants reveal the simultaneous formation of both long nanotubes and very small aggregates. The formation of very large molecular complexes is also confirmed by native PAGE. The observation of $\approx 10 \mu \mathrm{m}$-long tubes in the $24-8$ sample suggests that the tiles are able to arrange in a way to avoid steric clashes, perhaps by contorting the three-dimensional shape of the tile in order to form a more cooperative arrangement of connections with neighboring tiles. We observed the mean height of nanotubes formed from 23 to 7 is approximately $5.6 \pm 0.5 \mathrm{~nm}$, over $1 \mathrm{~nm}$ taller than $4.2 \pm 0.5$ $\mathrm{nm}$ tall nanotubes formed from 24 to 8 , suggesting that the diameter of 23-7 nanotubes is larger than the diameter of 24-8 nanotubes. Additionally, many of the 23-7 and 24-8 nanotubes appear chiral, based on the geometry of adjacent regions with distinct height that are defects in the tubular assembly; the observed chirality is consistent with the geometric effect we proposed for the offset in tile coaxial stacking caused by the RNA base pair tilt. Chirality is not observed in DNA DAE-E nanotubes, ${ }^{17}$ although spiral patterns can be programmed by tessellating distinct tile types. ${ }^{18,40}$

Continuing the structural series, variants 25-9 and 26-10 both formed exclusively linear fibers after annealing. These structures, in contrast to the nanotubes formed by 23-7 and $24-8$, are clearly predicted also by our model as a consequence of additive twist. In these cases, the excessive twist between adjacent tiles promotes assembly into one-dimensional fibers rather than networks. The observed height of these fibers $(\approx 1.5 \mathrm{~nm})$ is consistent with the height of the RNA double helix when probed by AFM. The characteristic smear observed in native PAGE is indicative of the presence of fibers with a smooth distribution of differing lengths varying by single tiling units. $^{30}$

AFM images reveal the presence of many 23-7 and 24-8 nanotubes whose length exceeds $10 \mu \mathrm{m}$. However, the physical limitations of measuring objects that are many microns long while only being nanometers tall make this measurement difficult to perform by AFM. Thus, we sought to image these samples using fluorescence microscopy to gather length statistics more rapidly. For this purpose, we purchased a Cy3 labeled variant of RNA strand S3, which was used to anneal both 23-7 and 24-8 nanotubes. (Only 10\% of the S3 strand was replaced with the labeled dye; nanotube yield was significantly reduced when using a higher concentration of labeled strand, as shown in Figure S9.) Example fluorescence microscopy images in Figure 3 show the presence of a large number of nanotubes. Formation of nanotubes was observed at tile concentrations above $250 \mathrm{nM}$ in both 23-7 and 24-8 variants (SI Section 5). With an automated image processing script, we identified RNA nanotubes and measured their length; a histogram of length data collected from 10 images taken after annealing is shown in Figure 3A,B,left. Many nanotubes reach $5-10 \mu \mathrm{m}$, but their mean length is $\approx 1.5 \mu \mathrm{m}$, less than half of the mean length generally observed in DAE-E DNA nanotubes. $^{17,31}$ 
We next examined the evolution of the RNA nanotube length distribution when nanotubes are stored at room temperature. It is well-known that DAE-E DNA nanotubes nucleate, polymerize, and join at room temperature from preannealed tiles. ${ }^{31}$ As a consequence, the mean length of DNA nanotubes continues to increase until it plateaus at around 30 $\mathrm{h}$ after annealing. ${ }^{31}$ We monitored the length distribution of 23-7 and 24-8 RNA nanotubes stored at room temperature for $30 \mathrm{~h}$ post-annealing, and we found that the mean nanotube length remains constant throughout the duration of the experiment (Figure S2). To clarify the reason why the nanotube mean length remains constant, we first asked whether nanotube joining occurs. For this purpose, we annealed a sample of 24-8 RNA nanotubes in which $10 \%$ of S3 strands are labeled with Atto-647N, and another sample in which $10 \%$ of S3 strands are labeled with Cy-3. Separately annealed $\mathrm{Cy}-3$ and Atto-labeled nanotubes were mixed and incubated at room temperature for $30 \mathrm{~h}$. Images indicate that nanotubes of distinct colors do not join, suggesting that joining is a rare event (Figure S3). To test whether nucleation and polymerization occur at room temperature, we designed a twotile variant of the 23-7 nanotubes; in this design assembly can occur only in the presence of both (pre-annealed) tiles, which interact with a diagonal pattern. ${ }^{17}$ Separately annealed tiles were mixed at room temperature, however no nanotubes were visible in fluorescence microscopy images (Figure S6), suggesting that nucleation and assembly at room temperature face a significant energy barrier. These experiments indicate that nucleation, polymerization, and joining of RNA nanotubes hardly proceed spontaneously at room temperature. We note that it is possible that the rate at which free tiles bind to formed nanotubes is comparable to the rate at which they unbind, resulting in the observation of a nearly constant mean nanotube length. However, the large number of very small lattices observed in AFM images for the 23-7 and 24-8 variants (Figure 2) suggests that most tiles may be trapped in these small lattices that then cannot contribute to tube polymerization.

Lastly, we examined the role of the sticky-end length in the formation of nanotubes. The energetics of tile-tile interactions are distinct in each of our variants, because in addition to changing the intertile crossover distance, we also varied the length of the sticky ends. Thus, we considered a 24-5 variant, in which the intertile crossover distance is 24 bases, but the sticky-end length is 5 bases (this variant required elongation of strands $S 1$ and S5, relative to the 24-8 tile characterized earlier). Because DAE-E DNA tiles by Rothemund et al. form nanotubes with a sticky-end length of 5 bases, we expected this length to be sufficient for RNA nanotube formaxtion; we also conjectured that shorter sticky ends would present less opportunities for nonspecific binding and secondary structure and that polymerization may occur at room temperature postannealing. Yet, this variant did not yield nanotubes (Figure S7). This may be due to a combination of different factors which have been previously observed to affect assembly of RNA tiles. ${ }^{7}$ These difficult-to-predict effects include tile-tile interactions that are too weak to compensate the twist-induced strain in the assemblies or even an unfavorable position of the nick along the intertile helices.

The fact that the length distribution of the 23-7 and 24-8 RNA nanotubes remains unchanged for over $30 \mathrm{~h}$ indicates that these structures are quite stable. The observed resilience of our RNA nanotubes makes them amenable to serve as scaffolds for other nucleic acids, proteins, or nanoparticles. DNA scaffolds, including DNA nanotubes, can be decorated with other components by introducing single-stranded domains that protrude from the scaffold and serve as binding sites for complementary oligonucleotides directly linked to the component of interest. Thus, we constructed a variant of the 23-7 tile that includes a 5-base long overhang at the $5^{\prime}$ end of strand S4. As exemplified in the fluorescence microscopy image in Figure $3 \mathrm{C}$, this tile variant assembled into nanotubes with a yield comparable to the original design, albeit with a reduced mean length $(\approx 0.3 \mu \mathrm{m})$.

To our knowledge, the RNA nanotubes described here are the largest existing structured RNA assemblies, with an average estimated size of 45-90 MDa. (The molecular weight of 23-7 and 24-8 tile variants is $60 \mathrm{kDa}$; the average nanotube length for the 23-7 and 24-8 tile variants is $\approx 1.5 \mu \mathrm{m}$, and individual tiles are $\approx 12 \mathrm{~nm}$ in length. We further assumed that the minimum estimated nanotube circumference is $\approx 6$ tiles and the maximum circumference is $\approx 12$ tiles.) These assemblies are much larger and have a higher yield than RNA lattices and tubular structures we characterized in a previous work. ${ }^{7}$ These earlier DAE-E tile variants account for the A-form helix geometry and are symmetric in regards to sequence, where the tile is composed of five strands, but only three have a unique sequence. The presence of repeated domains in the tiles may be one of the reasons behind the low yield and limited size of those assemblies (at most $\approx 1 \mu \mathrm{m}$ in length). ${ }^{32}$

An earlier demonstration of tile-based RNA nanotubes was described in Delebecque et al. ${ }^{33,34}$ The tiles were designed to be antiparallel with an odd-number of intra- and intertile halfturns between crossovers (DAO-O), yet the large base inclination of RNA was not taken into account in the designs. This issue was likely mitigated by the presence of unpaired nucleotides at the crossovers, which are expected to improve the tile flexibility. Designed interactions among RNA tiles were tested using split-GFP experiments, and with an assay to improve hydrogen metabolic production in vivo, yet it is difficult to ascertain the formation of structured assemblies based on the data available.

\section{CONCLUSIONS}

As other nucleic acid structures, our RNA nanotubes could serve as programmable scaffolds for other organic and nonorganic ligands. ${ }^{35,36}$ Because the inclusion of single-stranded overhangs to our tiles does not significantly affect the yield and mean length of nanotubes (Figure $3 \mathrm{C}$ ), we expect that these nanotubes could be decorated with aptamers, siRNA, and other RNA or DNA molecules of therapeutic value. ${ }^{35}$ An immediate application of these functional nanotubes could be the delivery of multiple nucleic acid therapeutics to cells, as recently shown with a comparable lattice design. ${ }^{8}$ In addition to providing the ability to control the stoichiometry of the therapeutic components, it has been shown that large scaffolds should also present a longer half-life in blood serum when compared to single-stranded or double-stranded RNA components. $^{8}$ Further, the linear RNA fibers assembling from tile variants $25-9$ and 26-10 (Figure 2D,E) may be engineered to deliver RNA therapeutic molecules with a reduced immunogenic response relative to other RNA nanoassemblies, as recently demonstrated for other programmable RNA fibers. ${ }^{37}$

Overall our results highlight that stable, large RNA structures can be readily obtained with the design principles 
imported from DNA nanotechnology. DX tiles are one of the most versatile motifs demonstrated by DNA nanotechnology and have been used to build a variety of algorithmic assemblies $^{38,39}$ as well as functional lattices and nanotubes, ${ }^{18,40}$ and our results indicate that DX RNA tiles could be used for similar purposes. As in DNA systems, the assembly of the RNA nanotubes presented here may be controlled precisely by taking advantage of other nucleic acid components; for example, DNA or RNA termination caps could be used to control the average nanotube size; ${ }^{41}$ temporal control of the assembly could be obtained by designing suitable strand invasion reactions on the tile sticky ends; ${ }^{27,28}$ and spatial control could be achieved by combining nanotubes with selfassembled nucleation sites. ${ }^{42,43}$

While RNA nanotechnology has made great advances in designing and building nanoscale RNA objects, lattices, and fibers, there has been slow progress toward building large and stable RNA structures that can be synthesized with a high yield. ${ }^{44}$ In contrast, the number of large, robust assemblies in DNA nanotechnology has been rapidly growing. ${ }^{45}$ By demonstrating that one of the most versatile tile motifs in DNA nanotechnology can be adapted to RNA and by highlighting the corresponding design challenges, we hope to accelerate the development of artificial, rationally programmable RNA components that will find applications in synthetic biology, materials science, and nanomedicine.

\section{METHODS}

Oligonucleotides. RNA strands were transcribed from DNA templates with a T7 promoter. DNA strands were purchased from Integrated DNA Technologies (Coralville, IA, USA). Concentrations of nucleic acids were determined by absorption measurements (Nanodrop 2000c, Thermo Scientific). DNA and RNA sequences are reported in SI Section 1.

RNA Extraction. Purified RNA strands were individually transcribed in vitro using the AmpliScribe T7-Flash transcription kit (\#ASF3507 Epicenter, Inc.) from corresponding DNA templates. RNA strands were gel extracted by either $10 \%$ or $15 \%$ denaturing polyacrylamide gel electrophoresis (PAGE). Next, RNA was eluted using $0.3 \mathrm{M}$ sodium acetate at $\mathrm{pH}$ 5.3. Finally, RNA was precipitated using ethanol and glycogen, dried, and resuspended in nuclease-free water.

Polyacrylamide Gel Electrophoresis (PAGE). Denaturing $P A G E$. A gel premix was prepared by mixing urea, nanopure water, acrylamide/bis-acrylamide 19:1, 40\% solution, which was heated until the urea completely dissolved. To start polymerization, the premix was mixed with Tris-borate-EDTA (TBE) buffer, ammonium persulfate (APS), and tetramethylethylenediamine (TEMED) and nanopure water in the appropriate amounts. The gels were allowed to polymerize for at least $2 \mathrm{~h}$ before electrophoresis. The gels ran at room temperature at $100 \mathrm{~V}$ in $1 \times \mathrm{TBE}$ unless otherwise noted. After electrophoresis, the gels were stained with SYBRGold Nucleic Acid Gel Stain (Invitrogen, \#S11494) for 20-30 min; gels were imaged using the Biorad ChemiDoc MP system.

Nondenaturing PAGE. Acrylamide/bis-acrylamide 19:1, 40\% solution, Tris-acetate-EDTA (TAE), magnesium chloride $\left(\mathrm{MgCl}_{2}\right)$, APS, and TEMED were added together at appropriate concentrations for the desired polyacrylamide percentage, then cast, and allowed to polymerize for at least $2 \mathrm{~h}$ before use. Gels ran at $4{ }^{\circ} \mathrm{C}$ at $150 \mathrm{~V}$ in $1 \times$ TBE buffer. After electrophoresis, the gels were stained with SYBRGold Nucleic Acid Gel Stain (Invitrogen, \#S11494) for 10 min and then imaged using the Biorad ChemiDoc MP system.

Atomic Force Microscopy. AFM images were obtained in tapping mode under buffer using a Digital Instruments Multimode AFM with a NanoscopeIII controller. We used either a Bruker SNL10 silicon tip on a nitride lever with a spring constant of $0.24 \mathrm{~N} / \mathrm{m}$ and a drive frequency of $9-10 \mathrm{kHz}$ or an Olympus BL-AC40TS-C2 with a spring constant of $0.09 \mathrm{~N} / \mathrm{m}$ and a drive frequency of $27-28$ $\mathrm{kHz}$. The AFM buffer consisted of the same buffer used for annealing unless otherwise noted.

Fluorescence Microscopy. Images were obtained with a NIKON TI-E inverted fluorescence microscope. A Cy3 filter was used to image all of the tiles with $\mathrm{Cy} 3$ labeled S3 strand. A $60 \times$ oil immersion objective was used to collect all images, with a standard exposure time of $600 \mathrm{~ms}$.

\section{ASSOCIATED CONTENT}

\section{S Supporting Information}

The Supporting Information is available free of charge on the ACS Publications website at DOI: 10.1021/acsnano.8b09421.

Additional experiments and further explanation (PDF)

\section{AUTHOR INFORMATION}

\section{Corresponding Author}

*E-mail: efranco@seas.ucla.edu.

ORCID $\odot$

Elisa Franco: 0000-0003-1103-2668

Notes

The authors declare no competing financial interest.

\section{ACKNOWLEDGMENTS}

The authors thank Paul W. K. Rothemund for useful discussions and advise. This work was supported by a U.S. National Science Foundation CAREER grant to E.F. [DMR1450747].

\section{REFERENCES}

(1) Fu, J.; Liu, M.; Liu, Y.; Woodbury, N. W.; Yan, H. Interenzyme Substrate Diffusion for an Enzyme Cascade Organized on Spatially Addressable DNA Nanostructures. J. Am. Chem. Soc. 2012, 134, $5516-5519$.

(2) Wilner, O. I.; Weizmann, Y.; Gill, R.; Lioubashevski, O.; Freeman, R.; Willner, I. Enzyme Cascades Activated on Topologically Programmed DNA Scaffolds. Nat. Nanotechnol. 2009, 4, 249-254.

(3) Erkelenz, M.; Kuo, C.-H.; Niemeyer, C. M. DNA-Mediated Assembly of Cytochrome P450 BM3 Subdomains. J. Am. Chem. Soc. 2011, 133, 16111-16118.

(4) Stewart, J. M.; Franco, E. Self-Assembly of Large RNA Structures: Learning from DNA Nanotechnology. DNA RNA Nanotechnol. 2016, 2, 23-35.

(5) Zuo, H.; Wu, S.; Li, M.; Li, Y.; Jiang, W.; Mao, C. A Case Study of the Likes and Dislikes of DNA and RNA in Self-Assembly. Angew. Chem. 2015, 127, 15333-15336.

(6) Geary, C.; Rothemund, P. W.; Andersen, E. S. A Single-Stranded Architecture for Cotranscriptional Folding of RNA Nanostructures. Science 2014, 345, 799-804.

(7) Stewart, J. M.; Subramanian, H. K.; Franco, E. Self-Assembly of Multi-Stranded RNA Motifs into Lattices and Tubular Structures. Nucleic Acids Res. 2017, 45, 5449-5457.

(8) Stewart, J. M.; Viard, M.; Subramanian, H. K.; Roark, B. K.; Afonin, K. A.; Franco, E. Programmable RNA Microstructures for Coordinated Delivery of siRNAs. Nanoscale 2016, 8, 17542-17550.

(9) Kawasaki, H.; Taira, K.; Wadhwa, R. World of Small RNAs: from Ribozymes to siRNA and miRNA. Differentiation 2004, 72, 58-64.

(10) Han, D.; Qi, X.; Myhrvold, C.; Wang, B.; Dai, M.; Jiang, S.; Bates, M.; Liu, Y.; An, B.; Zhang, F.; et al. Single-Stranded DNA and RNA Origami. Science 2017, 358, No. eaao2648.

(11) Linpinsel, J. L.; Conn, G. L. Recombinant and in Vitro RNA Synthesis; Springer: New York, 2013; pp 43-58.

(12) Milligan, J. F.; Groebe, D. R.; Witherell, G. W.; Uhlenbeck, O. C. Oligoribonucleotide Synthesis Using T7 RNA Polymerase and 
Synthetic DNA Templates. Nucleic Acids Res. 1987, 15 (21), 87838798.

(13) Davis, R. H. Large-Scale Oligoribonucleotide Production. Curr. Opin. Biotechnol. 1995, 6, 213-217.

(14) Marshall, W. S.; Kaiser, R. J. Recent Advances in the HighSpeed Solid Phase Synthesis of RNA. Curr. Opin. Chem. Biol. 2004, 8, 222-229.

(15) Chworos, A.; Severcan, I.; Koyfman, A. Y.; Weinkam, P.; Oroudjev, E.; Hansma, H. G.; Jaeger, L. Building Programmable Jigsaw Puzzles with RNA. Science 2004, 306, 2068-2072.

(16) Jaeger, L.; Chworos, A. The Architectonics of Programmable RNA and DNA Nanostructures. Curr. Opin. Struct. Biol. 2006, 16, 531-543.

(17) Rothemund, P. W.; Ekani-Nkodo, A.; Papadakis, N.; Kumar, A.; Fygenson, D. K.; Winfree, E. Design and Characterization of Programmable DNA Nanotubes. J. Am. Chem. Soc. 2004, 126, 16344-16352.

(18) Mitchell, J. C.; Harris, J. R.; Malo, J.; Bath, J.; Turberfield, A. J. Self-Assembly of Chiral DNA Nanotubes. J. Am. Chem. Soc. 2004, 126, 16342-16343.

(19) Douglas, S. M.; Chou, J. J.; Shih, W. M. DNA-NanotubeInduced Alignment of Membrane Proteins for NMR Structure Determination. Proc. Natl. Acad. Sci. U. S. A. 2007, 104, 6644-6648.

(20) Aldaye, F. A.; Lo, P. K.; Karam, P.; McLaughlin, C. K.; Cosa, G.; Sleiman, H. F. Modular Construction of DNA Nanotubes of Tunable Geometry and Single-or Double-Stranded Character. Nat. Nanotechnol. 2009, 4, 349.

(21) Shu, D.; Shu, Y.; Haque, F.; Abdelmawla, S.; Guo, P. Thermodynamically Stable RNA Three-Way Junction for Constructing Multifunctional Nanoparticles for Delivery of Therapeutics. Nat. Nanotechnol. 2011, 6, 658-667.

(22) Afonin, K. A.; Viard, M.; Koyfman, A. Y.; Martins, A. N.; Kasprzak, W. K.; Panigaj, M.; Desai, R.; Santhanam, A.; Grabow, W. W.; Jaeger, L.; et al. Multifunctional RNA Nanoparticles. Nano Lett. 2014, 14, 5662-5671.

(23) Geary, C.; Chworos, A.; Verzemnieks, E.; Voss, N. R.; Jaeger, L. Composing RNA Nanostructures from a Syntax of RNA Structural Modules. Nano Lett. 2017, 17, 7095-7101.

(24) Werner, C.; Krebs, B.; Keith, G.; Dirheimer, G. Specific Cleavages of Pure tRNAs by Plumbous Ions. Biochim. Biophys. Acta, Nucleic Acids Protein Synth. 1976, 432, 161-175.

(25) Jovine, L.; Djordjevic, S.; Rhodes, D. The Crystal Structure of Yeast Phenylalanine tRNA at 2.0 A Resolution: Cleavage by $\mathrm{Mg} 2+$ in 15-Year Old Crystals. J. Mol. Biol. 2000, 301, 401-414.

(26) Kurokawa, C.; Fujiwara, K.; Morita, M.; Kawamata, I.; Kawagishi, Y.; Sakai, A.; Murayama, Y.; Nomura, S.-i. M.; Murata, S.; Takinoue, M.; et al. DNA Cytoskeleton for Stabilizing Artificial Cells. Proc. Natl. Acad. Sci. U. S. A. 2017, 114, 7228-7233.

(27) Green, L. N.; Amodio, A.; Subramanian, H. K.; Ricci, F.; Franco, E. pH-Driven Reversible Self-Assembly of Micron-Scale DNA Scaffolds. Nano Lett. 2017, 17, 7283-7288.

(28) Green, L. N.; Subramanian, H. K.; Mardanlou, V.; Kim, J.; Hariadi, R. F.; Franco, E. Autonomous Dynamic Control of DNA Nanostructure Self-Assembly. Nat. Chem. 2019, submitted.

(29) Fu, T.; Seeman, N. DNA Double-Crossover Molecules. Biochemistry 1993, 32, 3211-3220.

(30) Nasalean, L.; Baudrey, S.; Leontis, N. B.; Jaeger, L. Controlling RNA Self-Assembly to Form Filaments. Nucleic Acids Res. 2006, 34, $1381-1392$.

(31) Ekani-Nkodo, A.; Kumar, A.; Fygenson, D. K. Joining and Scission in the Self-Assembly of Nanotubes from DNA Tiles. Phys. Rev. Lett. 2004, 93, 268301.

(32) Ko, S. H.; Su, M.; Zhang, C.; Ribbe, A. E.; Jiang, W.; Mao, C. Synergistic Self-Assembly of RNA and DNA Molecules. Nat. Chem. 2010, 2, 1050.

(33) Delebecque, C. J.; Lindner, A. B.; Silver, P. A.; Aldaye, F. A. Organization of Intracellular Reactions with Rationally Designed RNA Assemblies. Science 2011, 333, 470-474.
(34) Sachdeva, G.; Garg, A.; Godding, D.; Way, J. C.; Silver, P. A. In Vivo Co-Localization of Enzymes on RNA Scaffolds Increases Metabolic Production in a Geometrically Dependent Manner. Nucleic Acids Res. 2014, 42, 9493-9503.

(35) Afonin, K. A.; Grabow, W. W.; Walker, F. M.; Bindewald, E.; Dobrovolskaia, M. A.; Shapiro, B. A.; Jaeger, L. Design and SelfAssembly of siRNA-Functionalized RNA Nanoparticles for use in Automated Nanomedicine. Nat. Protoc. 2011, 6, 2022-2034.

(36) Koyfman, A. Y.; Braun, G.; Magonov, S.; Chworos, A.; Reich, N. O.; Jaeger, L. Controlled Spacing of Cationic Gold Nanoparticles by Nanocrown RNA. J. Am. Chem. Soc. 2005, 127, 11886-11887.

(37) Rackley, L.; Stewart, J. M.; Salotti, J.; Krokhotin, A.; Shah, A.; Halman, J. R.; Juneja, R.; Smollett, J.; Lee, L.; Roark, K.; et al. RNA Fibers as Optimized Nanoscaffolds for siRNA Coordination and Reduced Immunological Recognition. Adv. Funct. Mater. 2018, 28, 1805959.

(38) Rothemund, P. W.; Papadakis, N.; Winfree, E. Algorithmic SelfAssembly of DNA Sierpinski Triangles. PLoS Biol. 2004, 2, No. e424.

(39) Barish, R. D.; Schulman, R.; Rothemund, P. W.; Winfree, E. An Information-Bearing Seed for Nucleating Algorithmic Self-Assembly. Proc. Natl. Acad. Sci. U. S. A. 2009, 106, 6054-6059.

(40) Sharma, J.; Chhabra, R.; Cheng, A.; Brownell, J.; Liu, Y.; Yan, H. Control of Self-Assembly of DNA Tubules Through Integration of Gold Nanoparticles. Science 2009, 323, 112-116.

(41) Agrawal, D. K.; Jiang, R.; Reinhart, S.; Mohammed, A. M.; Jorgenson, T. D.; Schulman, R. Terminating DNA Tile Assembly with Nanostructured Caps. ACS Nano 2017, 11, 9770-9779.

(42) Mohammed, A. M.; Schulman, R. Directing Self-Assembly of DNA Nanotubes Using Programmable Seeds. Nano Lett. 2013, 13, 4006-4013.

(43) Mohammed, A. M.; Šulc, P.; Zenk, J.; Schulman, R. SelfAssembling DNA Nanotubes to Connect Molecular Landmarks. Nat. Nanotechnol. 2017, 12, 312.

(44) Li, H.; Lee, T.; Dziubla, T.; Pi, F.; Guo, S.; Xu, J.; Li, C.; Haque, F.; Liang, X.-J.; Guo, P. RNA as a Stable Polymer to Build Controllable and Defined Nanostructures for Material and Biomedical Applications. Nano Today 2015, 10, 631-655.

(45) Seeman, N. C.; Sleiman, H. F. DNA Nanotechnology. Nat. Rev. Mater. 2017, 3, 17068. 